\title{
Numerical computation of the domain of operation of an electrospray of a very viscous liquid
}

\author{
F. J. HI GUERA \\ E. T. S. Ingenieros Aeronáuticos, UPM. Plaza Cardenal Cisneros 3, 28040 Madrid, Spain
}

A numerical study is carried out of the injection of a very viscous liquid of small electrical conductivity at a constant flow rate through an orifice in a metallic plate under the action of an electric field. The conditions under which the injected liquid can form an elongated meniscus with a thin jet emanating from its tip are investigated by computing the flow, the electric field and the transport of electric charge in the meniscus and a leading region of the jet. A stationary solution is found only for values of the flow rate above a certain minimum. At moderate values of the applied field, this minimum flow rate decreases when the applied field or the conductivity of the liquid increase. The electric shear stress acting on the surface of the liquid is not able to drive the liquid into the jet at flow rates smaller than the minimum while, for any flow rate higher than the minimum, the transfer of electric current to the surface may occur in a slender region of the jet where charge relaxation effects are small and the field induced by the electric charge of the jet is important. At high values of the applied field, the flow rate must be higher than another minimum, which increases with the applied field, in order for the viscous stress to balance the strong electric stress acting on the meniscus. The two conditions taken together determine lower and upper bounds for the applied field at a given flow rate, but the value of the applied field at which a stationary jet is first established when this parameter is gradually increased is higher than the lower bound, leading to hysteresis. When the liquid is electrosprayed in a surrounding dielectric fluid, the viscous shear stress that this fluid exerts on the surface of the jet eventually balances the electric shear stress and stops the continuous stretching of the jet. A fraction of the conduction current is left in the jet when the effect of the outer liquid comes into play in the region where this current is transferred to the surface, and no stationary solution is found above a maximum flow rate that decreases when the viscosity of the outer liquid increases or the applied field decreases. Order of magnitude estimates of the electric current and the conditions in the current transfer region are worked out.

\section{Introduction}

The electrospray is a technique for generating nearly monodisperse sprays of small electrically charged drops of an electrically conducting liquid using electric stresses. A meniseus of the liquid to be sprayed is subject to an electric field by applying a high voltage between the liquid, which is in contact with an electrode, and another 
electrode at some distance from the meniscus. The electric field induces an electric current in the liquid that accumulates electric charge at its surface and causes an electric stress that elongates the meniscus in the direction of the field. Under different conditions, the meniscus may either shed charged drops or emit one or several jets that in turn break into drops. The different functioning modes of an electrospray and the transitions between them have been classified by Cloupeau \& Prunet-Foch (1990, 1994) and Jaworek \& Krupa (1999). The cone-jet mode (Cloupeau \& PrunetFoch 1989), in which the meniscus takes a conical shape with a single stationary jet emanating from its tip and breaking into drops at some distance downstream, is the most suitable for generating small monodisperse drops and has been extensively studied. In his pioneering work with a meniscus formed at the end of a capillary, Zeleny $(1915,1935)$ first showed that the balance of electric and surface tension stresses in the cone-jet mode requires a voltage of order $\left(\gamma a / \epsilon_{0}\right)^{1 / 2}$, where $\gamma$ is the surface tension of the liquid, $a$ is the radius of the base of the meniscus and $\epsilon_{0}$ is the permittivity of the surrounding medium. Taylor (1964) carried out a correct analysis of the balance of stresses using a spheroidal approximation for the shape of the meniscus in order to determine the onset voltage and showed that a conical meniscus of semiangle $\alpha=49.29^{\circ}$ is an exact hydrostatic solution (at least in the vicinity of the tip) for a special value of the voltage. Smith (1986) and Cloupeau \& Prunet-Foch (1989) found hysteresis, whereby the cone-jet can be maintained when the voltage is decreased below the onset voltage at which the cone-jet first appears when the voltage is gradually increased. Once a cone-jet is formed, the main parameters determining the size of the cone-to-jet transition region and the drops are the conductivity of the liquid and the flow rate injected into the meniscus. The size of the drops decreases when the flow rate decreases or the conductivity increases; see Fernández de la Mora \& Loscertales (1994), Chen \& Pui (1997) and Gañán-Calvo. Dávila \& Barrero (1997), among others, for scaling laws of the size of the drops and the electric current, and Barrero et al. (2004) for extensions of these results to cone-jets in baths of dielectric liquids. A stable cone-jet exists only within a range of values of the flow rate that shifts towards lower flow rates as the conductivity increases (Smith 1986).

Cloupeau \& Prunet-Foch (1989) experimentally determined the domain of operation or region of the voltage/flow rate plane where a cone-jet of a given liquid can be established. This region is bounded by a minimum voltage that depends on the flow rate and at which the system jumps to an oscillatory mode, and a maximum voltage above which instabilities, or a multiple-jet mode, or electrical discharges in the surrounding gas appear. As was already mentioned, the flow rate can be varied at constant voltage in the domain of operation between certain minimum and maximum values. Fernandez de la Mora (2007) noted that the existence of a maximum flow rate probably reflects that the surrounding gas becomes unable to pass the increasing flux of drops generated when the flow rate increases. The minimum flow rate determines the thinnest jet and the highest surface electric field that can be attained with a given liquid (Fernandez de la Mora \& Loscertales 1994). The breakup of the jet into drops is most regular near this minimum flow rate, leading to the smallest drops and the narrowest size distributions (Rosell-Llompart \& Fernández de la Mora 1994).

The origin of the minimum flow rate is not yet clear, despite the large amount of work devoted to ascertain it. Fernández de la Mora \& Loscertales (1994) experimentally found that the minimum flow rate is of order $\epsilon Q_{0}$ in many cases, where $\epsilon$ is the dielectric constant of the liquid and $Q_{0}=\epsilon_{0} \gamma / \rho K$, with $\rho$ and $K$ denoting the density and electrical conductivity of the liquid. According to these authors, 
charge relaxation effects appear in a certain region around the tip of the meniscus where the residence time of the flow is of the order of the electric relaxation time of the liquid $t_{e}=\epsilon \epsilon_{0} / K$, so that the electric charge of a material element of the liquid surface cannot increase at the same pace as the electric field acting on the surface element during its transit across the relaxation region. This condition determines the order of the electric current as a function of the flow rate. It also implies that the electric field due to the applied voltage enters the liquid in the relaxation region and leads to an electric shear stress that can drive the liquid into the jet. Fernandez de la Mora (2007) pointed out that the scaling laws for the length and radius of the jet derived by Cherney (1999) on the basis of these charge relaxation effects suggest that the jet could effectively disappear, or break up before the transfer of charge to its surface is complete, when the flow rate is of order $\epsilon Q_{0}$. As an alternative explanation, Guerrero et al. (2007) noted that the relaxation region is largely autonomous and is able to suck a flow rate of order $\epsilon Q_{0}$ or larger independently of the conditions of the meniscus, which may make a stationary configuration impossible if the meniscus is fed with a flow rate small compared to $\epsilon Q_{0}$. Gañan-Calvo et al. (1997) proposed that charge relaxation effects occur in a certain region of the jet, rather than around the tip of the meniscus, and obtained a minimum flow rate of order $\epsilon^{1 / 2} Q_{0}$ from this condition. Higuera (2008a) estimated the characteristic time of charging of the liquid surface in the region of the jet where the conduction current is transferred to the surface, showing that this time may be large compared to the electric relaxation time of the liquid.

In this paper, a model configuration is used to numerically investigate the domain of operation of a cone-jet of a very viscous liquid, for which the effect of the inertia can be neglected in the meniscus and in a leading region of the jet. The model differs from typical experiments in two important aspects. First, the disparity of scales between the radius of the jet and the base of the meniscus is not as large in the computations as in real experiments. As a consequence, some of the results depend on the size of the meniscus, while Fernández de la Mora \& Loscertales (1994) find that this size is irrelevant in their experiments and others. Second, a parallel plate electrode configuration is assumed, leading to a uniform electric field far from the meniscus instead of the more complex electric field around the metallic needle often used in experiments. The difference between the two fields is large at distances from the meniscus of the order of its size, but it is not expected to be essential around its tip, where the elongation of the meniscus intensifies the field in any case. The two differences between model and experiments commented here are required to make the computations affordable, but they may have an effect on the results that should be kept in mind when comparing them to the experiments reviewed above.

In the framework of this model, a minimum flow rate is found below which no stationary solution exists. At small values of the electric field applied to the meniscus, the minimum flow rate is determined by the failure of the electric shear stress to deflect the liquid surface and drive liquid into the jet against the restoring effect of the surface tension, which overcomes the normal electric stress when the minimum flow rate is approached. At high values of the applied field, the electric stresses are large and disrupt the meniscus if they are not balanced by large viscous stresses, which exist only for sufficiently high flow rates. Charge relaxation effects may be small in the vicinity of the minimum flow rate in both cases. A maximum flow rate is also found when the viscous force exerted on the jet by a surrounding fluid is taken into account. 


\section{Formulation}

A flow rate $Q$ of a liquid of viscosity $\mu$ and electrical conductivity $K$ (liquid 1 hereafter) is injected through a circular orifice of radius $a$ in a planar metallic plate into a region occupied by a dielectric liquid of viscosity $\lambda \mu$ (liquid 0 ) which is at rest far from the orifice. The permittivity of this liquid is $\epsilon_{0}$ and the permittivity of the liquid injected through the orifice is $\epsilon_{0} \epsilon$. The two liquids are immiscible with an interfacial surface tension $\gamma$. The metallic plate is set to a high voltage relative to another distant parallel plate, which leads to a uniform field $E_{i c}^{*}$ far from the orifice. This field induces a conduction current in liquid 1 that accumulates electric charge at its surface. The surface charge is convected by the flow, originating a surface current additional to the conduction current in liquid 1. In addition, the electric field and the surface charge cause an electric stress whose components normal and tangent to the surface (denoted $\tau_{n}^{e}$ and $\tau_{f}^{e}$ is what follows) are given in the right-hand sides of (2.3b) and (2.3c) (see Landau \& Lifshitz 1960; Saville 1997). In the conditions envisaged here, the electric stress strains the liquid into a jet of radius small compared to the radius $a$ of the injection orifice. The analysis of the evolution and conditions of existence of this jet is the subject of the present paper.

The Reynolds number $R e=\rho_{1} v_{c} a / \mu=\rho_{1} \gamma a / \mu^{2}$, where $\rho_{1}$ is the density of liquid 1 and $v_{c}=\gamma / \mu$ is a characteristic velocity determined by an order-of-magnitude balance of viscous and surface tension stresses, is assumed to be small. This Reynolds number is $1.8 \times 10^{-3}$ for glycerol in air and $7.4 \times 10^{-4}$ for glycerol in hexane when $a=0.5 \mathrm{~mm}$. The motion of the liquid in and around the meniscus and in at least a leading region of the jet is dominated by viscous forces and obeys the Stokes equations when $R e \ll 1$. The electric field in liquid $i$ (with $i=0,1$ ) is $\boldsymbol{E}_{i}=-\nabla \varphi_{i}$, where $\varphi_{i}$ is the electric potential in liquid $i$, which satisfies Laplace's equation. At the interface, the electric potential is continuous $\left(\varphi_{0}=\varphi_{1}\right)$ and the electric field satisfies $\epsilon_{0}\left(\boldsymbol{E}_{0}-\epsilon \boldsymbol{E}_{1}\right) \cdot \boldsymbol{n}=\sigma$ (Landau \& Lifshitz 1960). Here $n$ is a unit vector normal to the interface and pointing towards liquid 0 and $\sigma$ is the density of free surface charge. This $\sigma$ satisfies the transport equation $\mathrm{D} \sigma / \mathrm{D} t=K \boldsymbol{E}_{1} \cdot \boldsymbol{n}+\sigma \boldsymbol{n} \cdot \nabla \boldsymbol{v} \cdot \boldsymbol{n}$, where $\mathrm{D} \sigma / \mathrm{D} t=\partial \sigma / \partial t+v \cdot \nabla \sigma$ is the material derivative of $\sigma$ and $v$ is the velocity of the liquids at the interface. The first term on the right-hand side of the equation for $\sigma$ is the rate of accumulation of charge per unit area of the interface and unit time due to the component of the conduction current density in liquid $1, j=K E_{1}$, normal to the interface. The second term is the rate of change of $\sigma$ due to the stretching of the interface. Here $\boldsymbol{n} \cdot \nabla \boldsymbol{v} \cdot \boldsymbol{n}$ is the negative of the straining rate of a material element of the interface (Batchelor 1967). Conduction from liquid 1 tends to accumulate charge at the interface, but this charge reduces the normal electric field $\boldsymbol{E}_{1} \cdot \boldsymbol{n}$ that drives conduction (because $\boldsymbol{E}_{1} \cdot \boldsymbol{n}=\left(\boldsymbol{E}_{0} \cdot \boldsymbol{n}-\sigma / \epsilon_{0}\right) / \epsilon$ from the boundary condition for the electric fields normal to the interface). The characteristic time for conduction to screen liquid 1 from the outer field is the electric relaxation time $t_{e}=\epsilon_{0} \epsilon / K$, which follows from the balance $\sigma / t_{e} \sim K \boldsymbol{E}_{1} \cdot \boldsymbol{n}$ with $\sigma \sim \epsilon_{0} \boldsymbol{E}_{0} \cdot \boldsymbol{n} \sim \epsilon_{0} \epsilon \boldsymbol{E}_{1} \cdot \boldsymbol{n}$.

The mathematical formulation of the electrohydrodynamical problem is similar to that of (Higuera 2006,2007b,2008a) and will be described briefly here. Distances are scaled with the radius $a$ of the orifice, velocities with the viscous-capillary velocity $v_{c}=\gamma / \mu$, and electric fields with $E_{c}=\left(\gamma / \epsilon_{0} a\right)^{1 / 2}$. The electric potentials, density of surface charge and electric currents are scaled with $E_{c} a, \epsilon_{0} E_{c}$ and $\epsilon_{0} E_{c} v_{c} a$, respectively. The interface between the liquids, which is a free material surface, is denoted by $f(x, t)=0$. The governing equations and boundary conditions are (subscripts 0 and 
1 denote velocities, pressures and electric fields in liquids 0 and 1)

$$
\nabla \cdot v_{0}=0, \quad 0=-\nabla p_{0}+\lambda \nabla^{2} \boldsymbol{v}_{0}, \quad \nabla^{2} \varphi_{0}=0
$$

in liquid 0 , for $f(x, t)>0$,

$$
\nabla \cdot v_{1}=0, \quad 0=-\nabla p_{1}+\nabla^{2} v_{1}, \quad \nabla^{2} \varphi_{1}=0
$$

in liquid 1 , for $f(x, t)<0$,

$$
\begin{gathered}
\frac{\mathrm{D} f}{\mathrm{D} t}=0, \quad \boldsymbol{v}_{0}=\boldsymbol{v}_{1}, \\
p_{0}-p_{1}+\boldsymbol{n} \cdot\left(\tau_{1}^{\prime}-\tau_{0}^{\prime}\right) \cdot \boldsymbol{n}+\nabla \cdot \boldsymbol{n}=\frac{1}{2}\left(E_{0 n}^{2}-\epsilon E_{1 n}^{2}\right)+\frac{1}{2}(\epsilon-1) E_{1 t}^{2}, \\
\boldsymbol{t} \cdot\left(\tau_{1}^{\prime}-\tau_{0}^{\prime}\right) \cdot \boldsymbol{n}=\sigma E_{1 t}, \\
E_{0 n}-\epsilon E_{1 n}=\sigma, \quad E_{0 t}=E_{1 t}, \\
\frac{\mathrm{D} \sigma}{\mathrm{D} t}=\Lambda E_{1 n}+\sigma \boldsymbol{n} \cdot \nabla \boldsymbol{v} \cdot \boldsymbol{n}
\end{gathered}
$$

at the interface, $f(x, t)=0$, which is assumed to be anchored to the edge of the orifice $(f=0$ at the edge),

$$
\boldsymbol{v}_{0}=0, \quad \boldsymbol{v}_{1}=\frac{C a}{\pi} \boldsymbol{i}, \quad \varphi_{0}=\varphi_{1}=0
$$

at the metallic plate $(x=0)$, and

$$
p_{0}=p_{1}=0, \quad v_{0}=0, \quad \nabla \varphi_{0}=\nabla \varphi_{1}=-E_{\infty} i
$$

far from the plate. Here $p_{i}$, with $i=0,1$, is the pressure of liquid $i$ referred to the pressure of liquid 0 far from the orifice and scaled with $\mu v_{c} / a ; \tau_{0}^{\prime}=\lambda\left[\nabla v_{0}+\left(\nabla v_{0}\right)^{T}\right]$ and $\tau_{1}^{\prime}=\nabla \boldsymbol{v}_{1}+\left(\nabla \boldsymbol{v}_{1}\right)^{T}$ are the dimensionless viscous stress tensors; $\boldsymbol{n}$ and $\boldsymbol{t}$ are unit vectors normal and tangent to the interface; $E_{0 n}=E_{0} \cdot \boldsymbol{n}, E_{0 t}=E_{0} \cdot \boldsymbol{t}$, and similarly for $E_{1 n}$ and $E_{1 f} ; i$ is a unit vector perpendicular to the metallic plate, and $x$ is the dimensionless distance to the plate.

The five dimensionless parameters that appear in (2.1)-(2.5) are

$$
C a=\frac{\mu Q}{\gamma a^{2}}, \quad E_{\alpha:}=\frac{\epsilon_{0}^{1 / 2} a^{1 / 2} E_{i 0}^{*}}{\gamma^{1 / 2}}, \quad \epsilon, \quad \Lambda=\frac{\mu K a}{\epsilon_{0} \gamma}, \quad \lambda .
$$

The electric current $I$ induced by the applied field $E_{\infty}$ is the sum of the conduction current in the bulk of liquid 1 and the surface current due to the convection of the free electric charge at the interface. The sum of the two contributions to the current is a constant independent of $x$ for any stationary solution of (2.1)-(2.5). For an axisymmetric solution, $I=I_{b}+I_{s}$ with

$$
I_{b}=2 \pi \Lambda \int_{0}^{r_{s}} E_{1 x} r \mathrm{~d} r \quad \text { and } \quad I_{s}=2 \pi \sigma v_{s} r_{s}
$$

where $E_{1 x}$ is the axial component of the electric field in liquid $1, r$ is the distance to the symmetry axis, $r_{s}(x)$ is the radius of the cross-section of the interface and $v_{s}(x)$ is the velocity of the liquids at the interface.

The asymptotic form of the solution of (2.1)-(2.5) far from the orifice is very much affected by the presence of the outer liquid. In the absence of whipping or breakup, the stationary flow in the jet is quasi-unidirectional for $x \gg 1$. The Stokes equation 
in (2.2) can be integrated across the jet to yield, upon using the boundary conditions $(2.3 b)$ and $(2.3 c)$,

$$
\frac{\partial}{\partial x}\left(3 \pi r_{s}^{2} \frac{\partial v}{\partial x}\right)+\pi r_{s}^{2} \frac{\partial}{\partial x}\left(\tau_{n}^{e}+\tau_{n}^{0}-\frac{1}{r_{s}}\right)+2 \pi r_{s}\left(\tau_{i}^{e}+\tau_{t}^{0}\right)=0,
$$

which is a generalization of the result in Feng (2002) (see also Higuera 2006). Here $v=C a / \pi r_{s}^{2}$ is the velocity of liquid 1 , which is nearly uniform in the jet cross-section, and $\left(\tau_{n}^{e}, \tau_{t}^{\ell}\right)$ and $\left(\tau_{n}^{0}, \tau_{f}^{0}\right)$ are the components normal and tangent to the interface of the electric stress and the stress of liquid 0 on the interface. Far from the orifice $E_{1 t} \approx E_{\alpha}$. and the electric shear stress is $\tau_{f}^{e}=\sigma E_{1 f} \approx\left(I-I_{b}\right) E_{\alpha}, r_{s} / 2 C a$, where the density of surface charge has been obtained from the equality $I_{s}=I-I_{b}$ with $I_{s} \approx 2 \pi \sigma v r_{s}$, and $I_{b} \approx \pi \Lambda E_{\infty \circ} r_{s}^{2}$.

In the absence of outer liquid $(\lambda=0)$, it is $\partial \tau_{n}^{0} / \partial x=\tau_{t}^{0}=0$ in (2.8). The balance of the axial forces due to the effective axial viscous stress (first term of the equation), to the axial gradient of the pressure variation caused by the surface tension (in the second term), and to the electric shear stress (third term) gives in this case (Higuera 2006)

$$
r_{s} \approx\left(1+\sqrt{1+\frac{24}{\pi} I E_{\infty}}\right) \frac{C a}{2 I E_{\infty} x} .
$$

The radius of the jet, and therefore the conduction current, tend to zero far from the orifice.

When $\lambda>0$, the motion that the jet induces in the outer liquid can be represented as a line distribution of Stokeslets (Happel \& Brenner 1965). The condition of continuity of the velocity at the interface gives the viscous shear stress of the outer liquid on the interface as $\tau_{t}^{0}=-\lambda v / r_{s}$, up to logarithmically small terms. This stress eventually balances the electric shear stress $\left(\tau_{t}^{0} \approx-\tau_{t}^{e}\right)$, giving the algebraic equation

$$
\left(I-\pi \Lambda E_{x_{s}} r_{s}^{2}\right) r_{s}^{4}=\frac{2 \lambda C a^{2}}{\pi E_{\infty}}
$$

for the radius of the jet. The result that the radius of the jet tends to a constant asymptotic value far downstream is to be understood as a coarse approximation only, given the large error involved in the evaluation of $\tau_{t}^{0}$ above. This constant asymptotic radius is to be compared to the continuous decrease of the radius in (2.9). The conduction current tends to a constant value in the approximation leading to (2.10). This equation has two positive real roots if the parameter group $G=I^{3} / \lambda A^{2} E_{\alpha} C a^{2}>27 \pi / 2$ and none elsewhere. If $G$ is large, the conduction current is small compared to the total current for the lower root of $(2.10)$ and approximately equal to the total current for the upper root. The lower root, which is much smaller than the upper root and is probably the only one physically attainable in these conditions (see $\$ 3.2$ ), and the associated values of the velocity of the liquid and the density of surface charge, are

$$
r_{s} \approx r_{s i \infty}=\left(\frac{2 \lambda C a^{2}}{\pi E_{i \infty} I}\right)^{1 / 4}, \quad v \approx\left(\frac{E_{\infty} I}{2 \pi \lambda}\right)^{1 / 2}, \quad \sigma \approx\left(\frac{\lambda I^{3}}{8 \pi E_{i \infty} C a^{2}}\right)^{1 / 4} .
$$

Stationary axisymmetric solutions of (2.1)-(2.5) have been computed by letting the system evolve in time from an arbitrary initial condition. Boundary element methods have been used to solve the Laplace and Stokes equations (2.1) and (2.2), and a second order Runge-Kutta method has been used to integrate the evolution equations for the liquid interface and the density of surface charge in $(2.3 a)$ and $(2.3 e)$. As in 

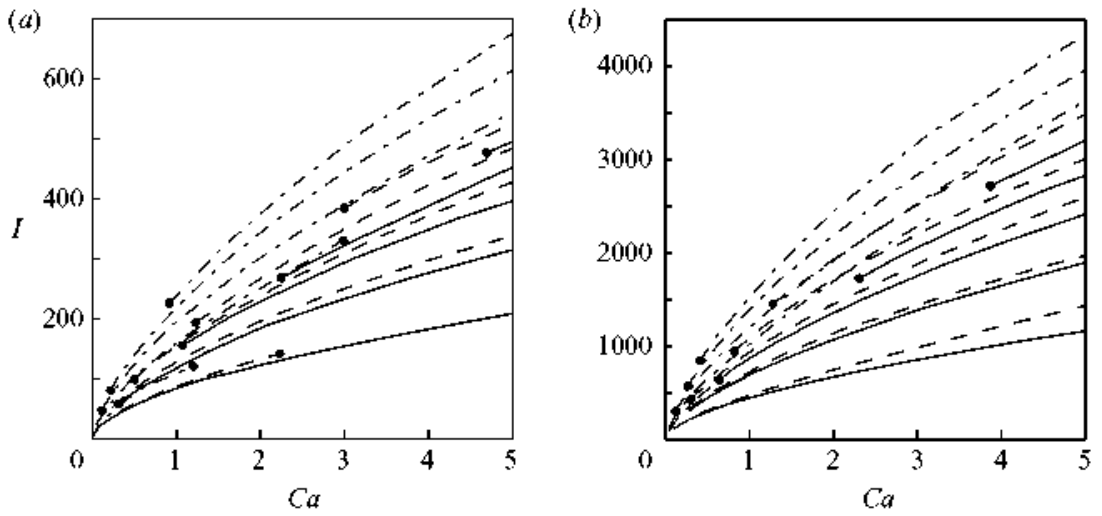

FIGURE 1. Dimensionless electric current $(\mathrm{I})$ as a function of the dimensionless flow rate $(\mathrm{Ca})$ for $\epsilon=20, \lambda=0$ (solid), 0.01 (dashed) and 0.1 (chain), $E_{c c}=1,2,3,4$ and 5 , increasing from bottom to top, and $A=10^{3}(a)$ and $2 \times 10^{4}(b)$. Some of the boundaries of the range of $C a$ where a solution exists are marked with dots.

previous works (Higuera 2006, 2007a, 2008a), the jet has been artificially truncated at a distance from the orifice sufficiently large to ensure, through numerical tests, that the solution is insensitive to the truncation.

\section{Results and discussion}

The electric current in shown in figure 1 as a function of the flow rate $\mathrm{Ca}$ for $\epsilon=20$, different values of the applied field $E_{\alpha}$ and the viscosity ratio $\lambda$, and two values of the dimensionless conductivity $\Lambda$. The electric current always increases with $C a$ and $E_{\infty}$. It also increases with $A$, though less than linearly, and with $\lambda$. The values $A=10^{3}$ and $2 \times 10^{4}$ used in figure 1 can be obtained for glycerol in air with $a=0.5 \mathrm{~mm}$ and conductivities $K=7.6 \times 10^{-7} \mathrm{~S} \mathrm{~m}^{-1}$ and $1.5 \times 10^{-5} \mathrm{~S} \mathrm{~m}^{-1}$.

There are several boundaries of the region of the parameter space where a stationary solution with a jet emanating from the tip of the meniscus of liquid 1 exists. There is a minimum flow rate below which no stationary solution is found. At low $E_{x,}$, the minimum flow rate decreases slightly when $E_{i \infty}$ increases (though this cannot be clearly seen at the scale of figure 1 ; see figures $2 a$ and 3) and the stationary solution breaks down in a leading region of the jet when the minimum flow rate is approached. At high $E_{\alpha}$, on the other hand, the electric forces are strong enough to disrupt the meniscus when the flow rate is too small, leading to a minimum flow rate that increases with $E_{\infty}$ (see figure 3 ).

In the presence of an outer liquid $(\lambda>0)$, there is also an upper limit to the flow rate, which increases with $E_{i \infty}$ and decreases when $\lambda$ increases. The electric shear at the interface is not able to overcome the viscous shear due to the outer liquid for values of the flow rate above this maximum. The stationary solution breaks down at the beginning of the jet when the maximum flow rate is approached.

The characteristics of the flow are analysed separately for $\lambda=0$ and $\lambda>0$ in what follows.

$$
\text { 3.1. Case of } \lambda=0
$$

\subsubsection{Minimum flow rate at hysteresis}

Figure 2(a) shows contours of constant volume of the meniscus in a region of the ( $E_{\alpha,}, C a$ ) plane for $\epsilon=20, \lambda=0$ and $A=10^{3}$ (solid contours) and $2 \times 10^{4}$ (dashed 

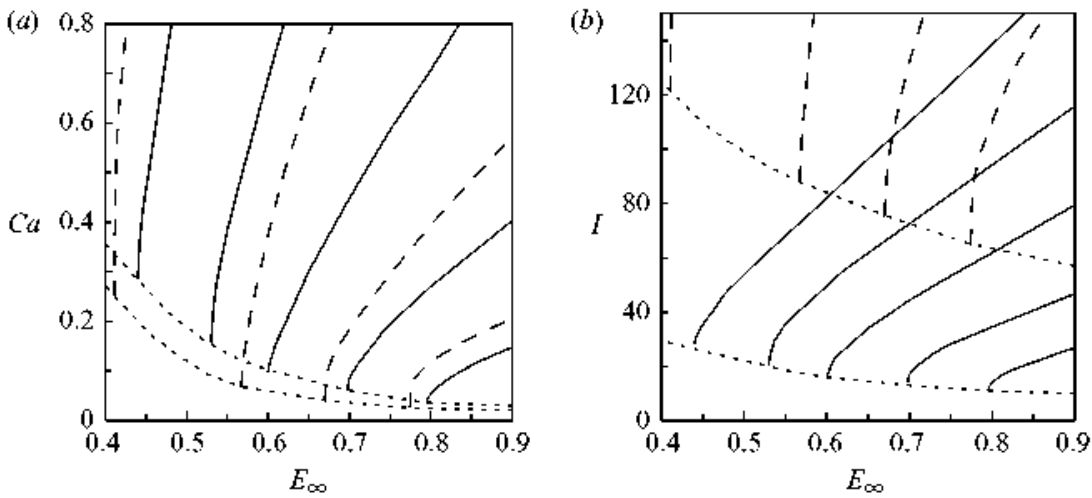

Figure 2. Contours of constant volume in the electric field/flow rate plane (a) and the electric field/electric current plane $(b)$ for $\epsilon=20, A=10^{3}$ (solid contours) and $2 \times 10^{4}$ (dashed contours). Dotted curves give the minimum flow rate (in $(a)$ ) and the minimum electric current (in $(b)$ ) as functions of the electric field for each value of $A$. Contours shown are, from bottom-right to top-left, $V=0.7,0.9,1.2,1.5$ and 2 for $A=10^{3}$, and $V=0.7,0.9,1.2$ and 2 for $A=2 \times 10^{4}$, where $V$ is the volume of liquid in the meniscus scaled with $a^{3}$.

contours). Stationary solutions with a given volume of liquid in the meniscus exist only above a certain $E_{x}$ at which the corresponding contour in figure $2(a)$ becomes vertical. The minimum $E_{c 0}$ decreases, and the associated minimum $C a$ increases, when the volume of liquid increases. The locus of these minima (dotted curves in figure $2 a$, for each of the two values of $A$ considered) defines the first boundary of the region of existence of stationary solutions mentioned above. In the operation of an electrospray at constant $E_{\alpha,}$ the domain is traversed downwards along a vertical line when the dimensionless flow rate $\mathrm{Ca}$ decreases. The volume of the meniscus decreases with $\mathrm{Ca}$ until the lower boundary (minimum $C a$ and volume for the value of $E_{\infty}$ chosen) is reached. The electric current emitted by the jet is shown in figure $2(b)$.

It may be noted that a hydrostatic meniscus $(C a=0)$ with a given volume of liquid attached to a metallic plate exists when $E_{\infty}$ is smaller than a certain $E_{\infty, H}$ that also decreases when the volume of the meniscus increases (see e.g. Higuera 2008b). For a given volume, this $E_{x_{S H}}$ is larger than the minimum $E_{\infty}$ in figure 2(a). For example, it is $E_{x_{H H}} \approx 0.86$ for $V=0.9$ and $E_{x_{H H}} \approx 0.56$ for $V=2$, where $V$ is the volume of liquid scaled with $a^{3}$. Therefore there is a range of $E_{\infty}$ where two stable solutions are possible for a meniscus of a given volume; a hydrostatic solution with a rounded surface and a meniscus that emits a thin jet from its tip, as in figure $4(a)$. (The second solution, of course, requires a continuous supply of liquid to make for the flow rate emitted by the jet and keep the volume constant). This multiplicity leads to hysteresis, as will be discussed below.

In addition to the two stable solutions, there is also an unstable hydrostatic solution for the same volume of liquid when $E_{\infty}$ is in a range that extends from $E_{x_{S H}}$ to a smaller value $E_{i \circ C}<E_{x_{M H}}$ (see e.g. Taylor 1964; Miksis 1981; Wohlhuter \& Basaran 1992). The origin of this branch of unstable hydrostatic solutions is in the intensification of the electric field and the electric stress at the surface when the meniscus elongates under the action of the electric stress. The elongation of the unstable meniscus increases when $E_{\alpha}$ decreases, until the meniscus develops a conical

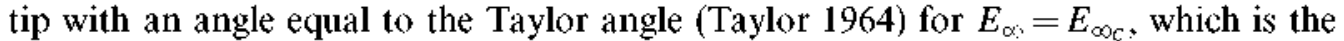
end point of the unstable branch. The shape of this hydrostatic pointed meniscus has 
been computed by Pantano, Gañán-Calvo \& Barrero (1994) for different values of the volume of liquid in the usual configuration where the meniscus is formed at the tip of a capillary, but similar results should be expected for a meniscus attached to a metallic plate.

It has been proposed (Pantano et al. 1994; Fernández de la Mora 2007) that the meniscus of a cone-jet tends to this pointed hydrostatic meniscus when the minimum flow rate is approached. This proposal is based on the grounds that (i) the pressure variation and viscous stresses due to the motion of the liquid are small compared to the electric and surface tension stresses on most of the surface of the liquid when the flow rate is small; and (ii) the electric field induced at the meniscus by the electric charge of the jet is small. If these two conditions are satisfied, the electric stress is determined by the shape of the meniscus only, and the equilibrium of electric and surface tension stresses (plus a nearly uniform pressure of the liquid) is not be affected by the flow in the meniscus or the presence of a jet. The finding of Fernandez de la Mora (1992), that cone angles very close to Taylor's are formed near the minimum flow rate, gives strong support to this proposal.

In the present configuration of a meniscus attached to a metallic plate, the hydrostatic pointed meniscus analogous to that of Pantano et al. (1994) would be realized at the lower boundary in figure $2(a)$, and the value of $E_{\infty}$ at which the contour in this figure for a given volume of liquid becomes vertical would coincide with $E_{\infty}$. In these conditions, the minimum value of $E_{\infty}$ in figure 2(a) for a given volume of liquid should be independent of $A$, because this parameter does not enter the analysis of the hydrostatic meniscus. This is not confirmed by the numerical results; inspection of the numerical solutions shows that the charge per unit length of the jet depends on the dimensionless conductivity $A$, and the electric field induced by this charge on the meniscus is not entirely negligible for the values of $A$ used in the computations. The result, however, could be peculiar to liquids of low conductivity. The estimates of $\$ 3.1 .2$ suggest that, at the minimum flow rate, the charge per unit length of the jet and the length of the current transfer region decrease when the conductivity of the liquid increases. This tends to bring the limiting shape of the meniscus at the lower boundary in figure 2(a) closer to the hydrostatic shape for liquids of high conductivity. But, since the hydrostatic meniscus is unstable, it is not clear how closely it can be approached, by decreasing the flow rate and therefore the effect of the charge of the jet, before the instability develops. This instability could set a different lower bound to the flow rate for liquids of high conductivity. The insets in figure 5 below show that the limiting meniscus may resemble a Taylor cone already for the moderate conductivities considered here.

Figure 3 shows a more complete picture of the domain of operation of a cone-jet. It includes an upper $E_{i \infty}$ boundary, at the right-hand side of the figure, in addition to the lower boundary discussed above. The electric stresses at the surface increase with $E_{\infty}$ and disrupt the meniscus when the upper boundary is crossed. Below this boundary, the electric stresses are balanced by pressure and viscous stresses, whose strength increases with the flow rate $\mathrm{Ca}$. For this reason, the minimum $\mathrm{Ca}$ at which a stationary solution ceases to exist increases with $E_{\infty ;}$ on this upper boundary. The insets at the right-hand side of the figure illustrate the disruption of the meniscus when the upper boundary is crossed. The meniscus does not resemble a Taylor cone near this boundary. Moreover, since the condition of axisymmetry used in the computations needs not be satisfied in reality, a transition to a multiple-jet mode may occur for a value of $E_{i c}$ smaller than the upper boundary. Figure 3 also shows that the volume of the meniscus decreases when $E_{\infty}$ increases at constant flow rate, 


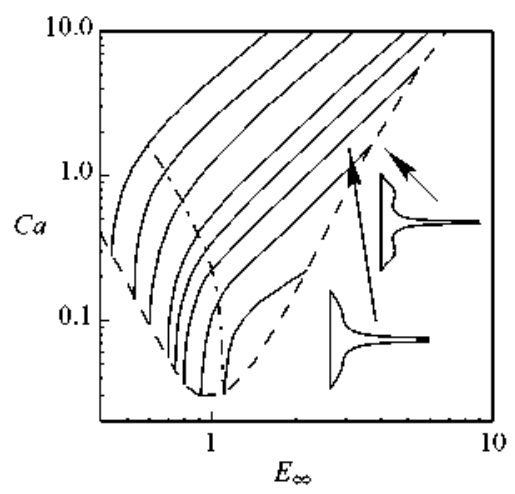

FIGURE 3. Contours of constant volume in the electric field/flow rate plane for $\epsilon=20, A=10^{3}$ and $\lambda=0$. Contours shown (solid curves) are $V=0.5,0.6,0.7,0.8,0.9,1.2,1.5$ and 2 , increasing from bottom to top. The dashed curves give the minimum and maximum values $E_{\infty}$ between which a stationary solution exists as functions of the flow rate $C a$. The chain curve gives the value of $E_{\infty}$ at which a cone-jet is first established when this parameter is gradually increased at constant flow rate.

which is in agreement with experimental results (Hayati, Bailey \& Tadros 1987; Cloupeau \& Prunet-Foch 1989). The radius of the jet in the current transfer region (not shown) follows the same trend. The dash-and-dot curve in figure 3 shows $E_{\infty_{H}}$ for different values of the volume of the meniscus. Since no hydrostatic solution exists above $E_{\alpha_{H}}$, this curve gives the value of $E_{\alpha}$ at which a cone-jet first appears when $E_{i \circ}$ is gradually increased, while the dashed curve at the left-hand side of the figure gives the value of $E_{x_{i}}$ at which the cone-jet disappears when $E_{\infty}$ is gradually decreased.

\subsubsection{Current transfer region and breakdown at the lower $E_{\infty}$ boundary}

Figure 4 shows different magnitudes pertaining to the solution of $(2.1)-(2.5)$ as functions of the distance $x$ from the metallic plate for $A=2 \times 10^{4}, \epsilon=20, \lambda=0, V=1.2$, and the two values of the applied field $E_{\infty}=0.568$ (which is about the minimum $E_{i 0}$ for this value of the volume; see figure $2 a$ ), and $E_{i 0}=0.70$. The corresponding values of the dimensionless flow rate are $C a=0.111$ and 0.877 , respectively. As can be seen in figure $4(b)$, there is a cross-over point where the conduction and convection contributions to the electric are equal to each other. This occurs at $x \approx 2$ in the first case and at $x \approx 3$ in the second. The components of the electric field normal and tangent to the surface (not displayed) have maxima near this cross-over point.

In the two cases shown in figure 4, the current transfer region where convection of the surface charge takes over from conduction is well into the jet, where the flow is quasi-unidirectional and (2.8) applies. To further check this result, the three terms of (2.8) (with $\partial \tau_{n}^{0} / \partial x=\tau_{t}^{0}=0$ ) have been evaluated using the full numerical solution of $(2.1)-(2.5)$ with the velocity at the symmetry axis playing the role of $v$. The results are shown in figure $4(c)$. The sum of the three terms (dotted curves), which would be zero if (2.8) were satisfied exactly, gives an idea of the error involved in the approximation of quasi-unidirectional flow. As can be seen, this error is small in the current transfer region, where the viscous and electric forces (first and third terms of (2.8); dashed and solid curves in figure $4 \mathrm{c}$ ) are the major axial forces. The estimates worked out elsewhere for a slender current transfer region (Higuera 2006, 2008a) are therefore 
(a)
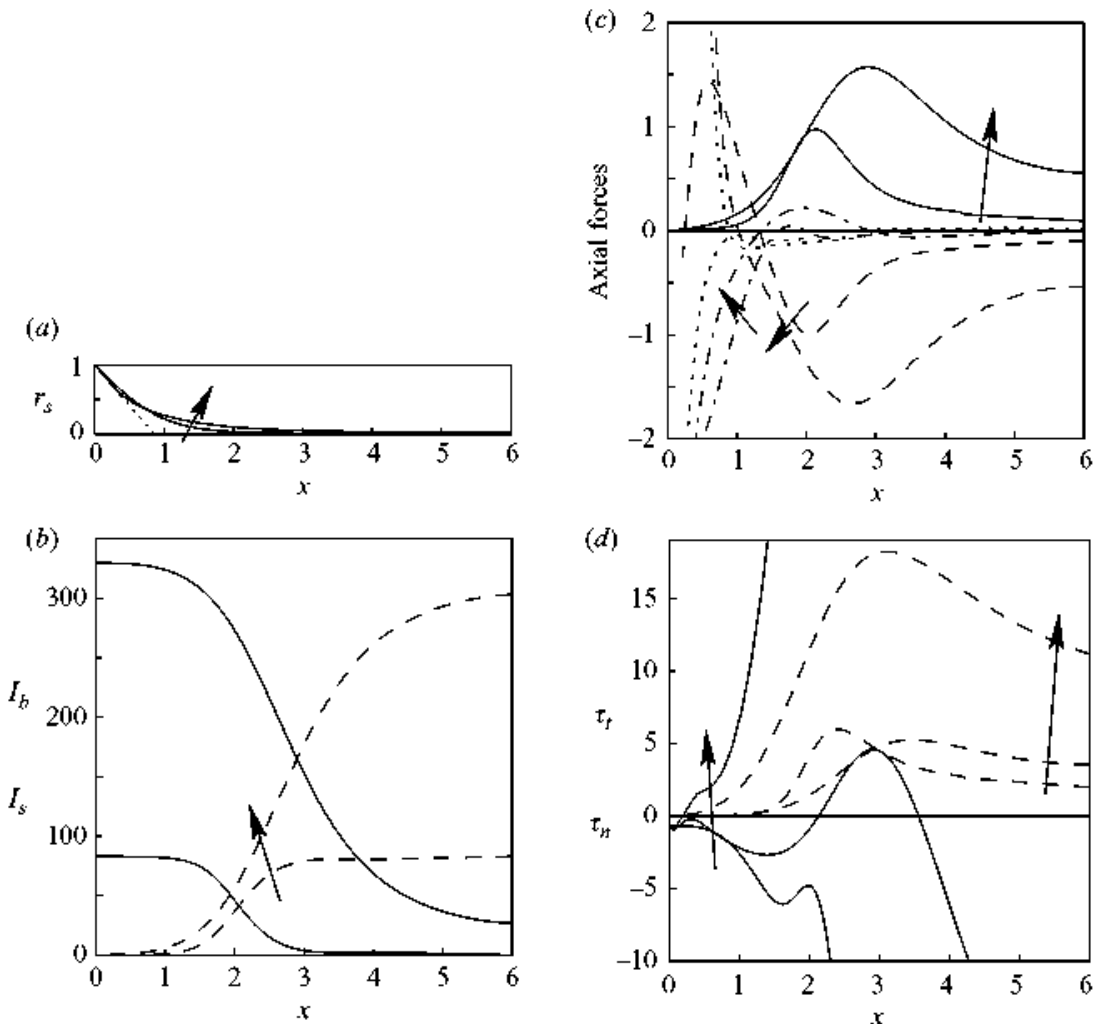

FIgure 4. (a) Radius of the cross-section of the surface, (b) conduction (solid) and convection (dashed) contributions to the electric current, (c) axial forces acting on the liquid per unit streamwise length (electric force, $2 \pi r_{s} \tau_{f}^{*}$ in the last term of (2.8) (solid); viscous force, first term of (2.8) (dashed); force due to the normal electric stress and the surface tension, $\pi r_{s}^{-3} \partial\left(\tau_{n}^{\ell}-1 / r_{s}\right) / \partial x$ in the second term of (2.8) (chain) and sum of these three forces (dotted)) and $(d)$ stresses normal $\left(\tau_{n}=\tau_{n}^{t}-\nabla \cdot n\right.$, solid) and tangent $\left(\tau_{t}=\tau_{t}^{\epsilon}\right.$, dashed) to the surface as functions of the distance $x$ to the plate for $V=1.2, \epsilon=20, A=2 \times 10^{4}, \lambda=0$, and $E_{\dot{\omega}}=0.568$ and 0.7 (in $(a)-(c)$ ), and $0.568,0.7$ and 2 (in $(d)$ ), increasing as indicated by the arrows. The dotted line in $(a)$ has the slope of a Taylor cone.

valid down to nearly the minimum $E_{\alpha}$ and $\mathrm{Ca}$ in figure $2(a)$. Briefly summarized, these estimates give the electric current, the radius of the jet in the current transfer region and the characteristic length of this region as

$$
I \sim \Lambda^{1 / 2} E_{i \infty}^{2 / 3} C a^{2 / 3}, \quad r_{s} \sim r_{s T}=\frac{C a^{1 / 3}}{\Lambda^{1 / 4} E_{\infty}^{1 / 6}}, \quad \ell \sim \frac{C a^{1 / 3}}{E_{\infty}^{2 / 3}}
$$

for $A^{1 / 2} / E_{i c} \gg \epsilon$. The results (3.1) follow from the conditions that (i) the conduction and convection contributions to the current are of the same order $\left(\Lambda E_{\infty \infty} r_{s}^{2} \sim \sigma v r_{s}\right.$, with $v \sim C a / r_{s}^{2}$ ); (ii) the axial field induced by the electric charge of the jet, which acts as a line of charge, partially balances the applied field $\left(\sigma r_{s} / \ell \sim E_{\alpha}\right.$; see e.g. Ashley \& Landahl 1965; Hinch 1991) and (iii) the axial electric force acting on the surface $\left(2 \pi r_{s} \tau_{t}^{e}\right.$ in $\left.(2.8)\right)$ is of the same order as the axial viscous force (first term of $\left.(2.8)\right)$. which gives $r_{s}^{2} v / \ell^{2} \sim r_{s} \sigma E_{\infty}$. In dimensional variables, denoted with asterisks, the 


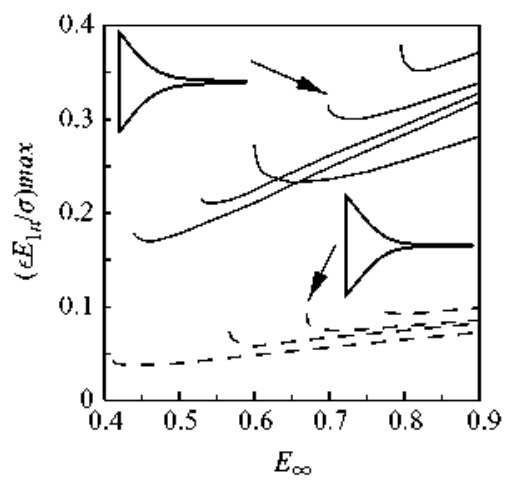

FIGURE 5. Maximum value of $\epsilon E_{1 n} / \sigma$ on the surface of the liquid as a function of $E_{\omega}$ for $\epsilon=20, A=10^{3}$ (solid) and $2 \times 10^{4}$ (dashed), $\lambda=0$ and $V=0.7,0.9,1.2,1.5$ and 2 for $A=10^{3}$ and $0.7,0.9,1.2$ and 2 for $A=2 \times 10^{4}$, increasing from right to left. The insets show the contours of the menisci at minimum $E_{\infty}$ for $V=0.9$.

estimates (3.1) read

$$
I^{*} \sim \epsilon_{0}^{1 / 3} \mu^{1 / 6} K^{1 / 2} E_{\infty}^{*^{2 / 3}} Q^{2 / 3}, \quad r_{s_{T}}^{*}=\frac{\epsilon_{0}^{1 / 6} \mu^{1 / 12} Q^{1 / 3}}{K^{1 / 4} E_{\infty}^{* 1 / 6}}, \quad \ell^{*} \sim \frac{\mu^{1 / 3} Q^{1 / 3}}{E_{\infty}^{* 2 / 3}}
$$

for $\mu^{1 / 2} K^{1 / 2} / \epsilon_{0} E_{\infty}^{*} \gg \epsilon$, which are independent of the radius of the orifice $a$.

According to (3.1), the ratio of the electric relaxation time $\left(t_{e}=\epsilon / \Lambda\right.$ in dimensionless variables) to the residence time of the flow in the current transfer region $\left(t_{r}=\ell / v \sim \ell r_{s}^{2} / C a\right)$ is $t_{e} / t_{r} \sim \epsilon E_{\infty} / \Lambda^{1 / 2} \ll 1$ in the conditions when (3.1) applies. Electric charge has plenty of time to accumulate at the surface and screen the liquid from the outer normal field $E_{0 n}$ during the transit of the liquid across the current transfer region. Therefore charge relaxation effects are not expected to play an important role when $\epsilon E_{\alpha} / \Lambda^{1 / 2}$ is small. This is confirmed in figure 5, where the maximum value of $\epsilon E_{1 n} / \sigma$ is small for $A=2 \times 10^{4}$ (dashed curves), for which $\epsilon E_{00} / \Lambda^{1 / 2}$ is of the order of 0.1 in the conditions of the figure, and moderate for $\Lambda=10^{3}$ (solid curves), for which $\epsilon E_{\infty} / \Lambda^{1 / 2}$ is about 4.5 times larger. In both cases, $\epsilon E_{1 n} / \sigma$ increases with $E_{\infty}$. except in the vicinity of the minimum $E_{i 0}$. Notice that $\epsilon E_{i \infty} / \Lambda^{1 / 2}=\epsilon \epsilon_{0} E_{c 0}^{*} / \mu^{1 / 2} K^{1 / 2}$ does not depend on the radius of the orifice.

The slender current transfer region discussed here is quite different from the current transfer region around the tip of a conical meniscus analysed by Fernández de la Mora \& Loscertales (1994), where charge relaxation effects are necessarily important. For the moderate conductivities considered in this paper, the numerical results show that charge relaxation effects are not important insofar as $\epsilon E_{\infty} / \Lambda^{1 / 2}$ is small. However, the situation may be more complex for liquids of high conductivity or larger injection orifices, for which the dimensionless radius of the jet is very small and the electric field acting on the jet is of the order of the electric field of a Taylor cone in a wide region downstream of the apparent apex of the meniscus. The balances leading to (3.1), which rely on the assumption that the electric field is of order $E_{i 0}$, should be modified when the transfer of current to the surface occurs in this region. Replacing $E_{i 0}^{*}$ in (3.2) by the field of a Taylor cone at a distance $\ell^{*}$ from its apex, $E_{T} \sim\left(\gamma / \epsilon_{0} \ell^{*}\right)^{1 / 2}$, these estimates transform into the well-known alternative estimates

$$
I^{*} \sim \gamma^{1 / 2} K^{1 / 2} Q^{1 / 2}, \quad r_{s_{T}}^{*} \sim \frac{\epsilon_{0}^{1 / 4} \mu^{1 / 8} Q^{3 / 8}}{\gamma^{1 / 8} K^{1 / 4}}, \quad \ell^{*} \sim \frac{\mu^{1 / 2} Q^{1 / 2}}{\gamma^{1 / 2}},
$$


which are applicable with the restrictions noted in Higuera (2003) and could provide a connection with the charge relaxation region of Fernández de la Mora \& Loscertales (1994). In particular, analysis of this important case should clarify whether the current transfer region still extends into the jet at the minimum flow rate. Unfortunately, this analysis is beyond the scope of the numerical method used here, due to the disparity of the jet and meniscus scales.

Figure $4(d)$ shows the normal and tangent stresses acting on the surface of the liquid for three values of $E_{\infty}$. The net normal stress is the difference between the outward electric stress $\tau_{n}^{e}$ and the inward surface tension stress $\nabla \cdot n$. The tangent stress is $\tau_{f}^{e}$. At the largest value of $E_{\infty}$ in figure $4(d)$, the normal stress is positive (points away from the liquid) in the whole meniscus and an ample region of the jet. pulling the liquid into the jet in combination with the electric shear stress. As $E_{\infty}$ decreases keeping the volume of liquid constant, the normal stress becomes negative in the meniscus and takes positive values only in a limited region of the jet. This region disappears, leaving a normal stress that points everywhere towards the liquid, when $E_{i \circ}$ approaches its minimum value. In these conditions, the motion of the liquid towards the tip of the meniscus and into the jet is only due to the electric shear stress, with the normal stress inducing a pressure gradient that pushes the liquid in the streamwise direction only within the region of the jet where $\partial\left(\tau_{n}^{e}-1 / r_{s}\right) / \partial x>0$ (see (2.8)).

The flow rate that the electric shear stress can drive into the jet decreases with $E_{\infty}$, despite the intensification of the field around the tip of the meniscus due to the decrease of the jet radius, which causes the peak value of $\tau_{f}^{e}$ in figure $4(d)$ to increase when $E_{s \circ}$ decreases in the vicinity of its minimum. Apparently the electric shear stress cannot overcome the inward normal stress when $E_{x_{i}}$ falls below the minimum in figure $2(a)$, and a stationary solution ceases to exist.

Numerical simulations of the evolution following a step decrease of $E_{i 0}$ from a value slightly above this minimum to a value slightly below show that the jet collapses at a point not far from the meniscus and the tip of the meniscus subsequently recedes and becomes blunt.

The electric shear stress is always small in the meniscus. The normal stress is nearly uniform in the meniscus at the smallest value of $E_{\alpha}$ in figure $4(d)$, which means that the flow is weak and the normal stress is balanced by a nearly uniform pressure of the liquid. When $E_{i 0}$ increases, the flow in the meniscus intensifies and the normal stress ceases to be uniform.

In orders of magnitude, the ratio of surface tension to normal electric stress in the current transfer region is $\left(1 / r_{s}\right) / \tau_{n}^{e} \sim 1 /\left(\Lambda^{1 / 4} E_{\alpha_{i}}^{5 / 6} \mathrm{Ca}^{1 / 3}\right)$, where use has been made of (3.1) to estimate the normal electric stress as $\tau_{n}^{e} \sim \sigma^{2} \sim\left(I r_{s T} / C a\right)^{2}$. This ratio is small if $C a \gg 1 /\left(\Lambda^{3 / 4} E_{x_{i}}^{5 / 2}\right)$ (or $Q \gg \gamma^{3} /\left(\epsilon_{0} \mu^{7 / 4} K^{3 / 4} E_{i \infty}^{* 5 / 2}\right)$, independent of $a$, in dimensional variables), whereas the effect of the surface tension comes into play and may lead to the capillary breakup of the jet before the transfer of current to its surface is complete if this condition is not satisfied. This coarse estimation suggests a minimum flow rate that decreases when the electric field or the electrical conductivity of the liquid increase, in qualitative agreement with the trends of the numerical results in figure 2(a). Similar arguments have been used by Kim \& Turnbull (1976) and Larriba \& Fernández de la Mora (2009) for a related problem.

\subsection{Case of $\lambda>0$}

Figure 6(a) shows longitudinal sections of the interface for $\lambda=0,0.01$ and 0.1 and constant values of other parameters. The radius of the jet increases with $\lambda$ and does 

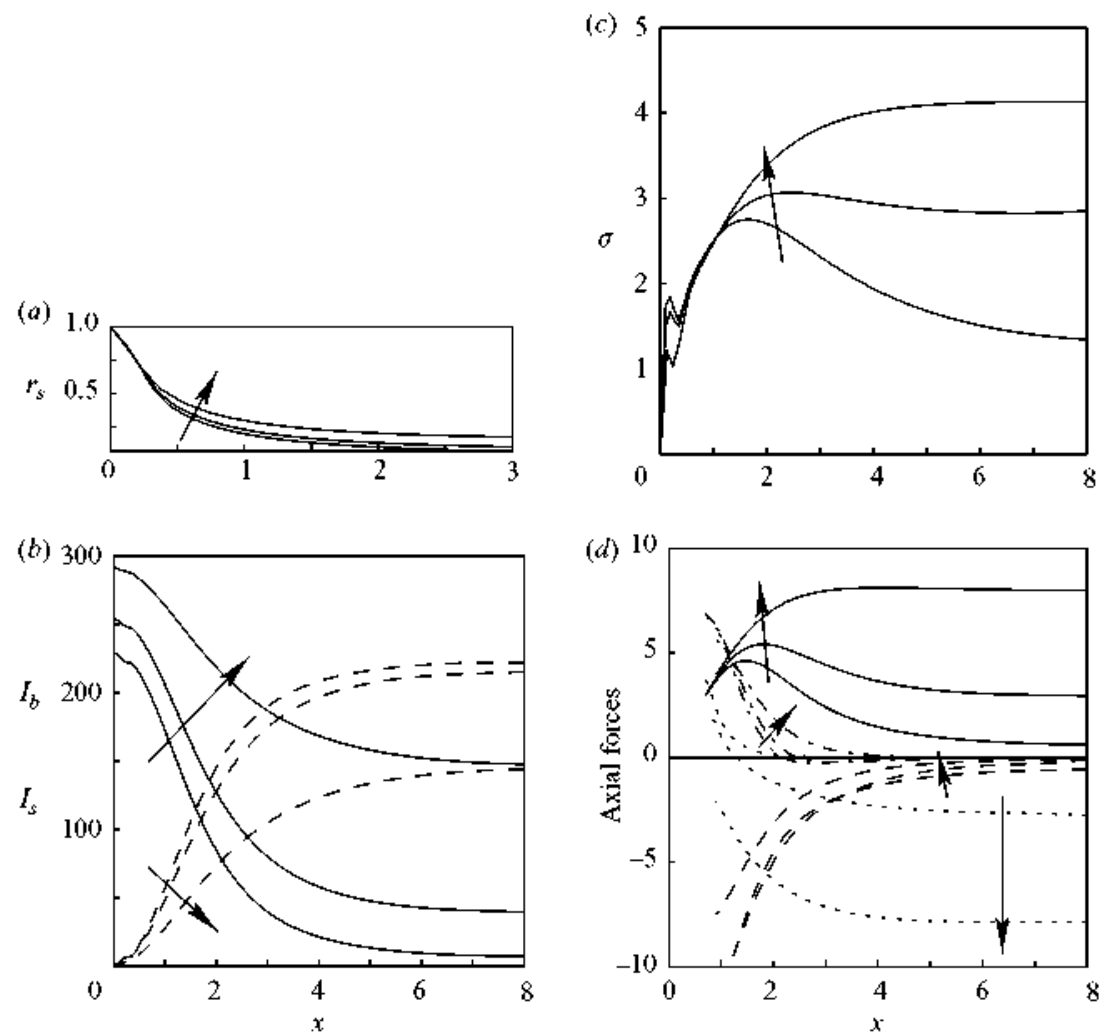

Figure 6. (a) Radius of the cross-section of the surface, (b) conduction (solid) and convection (dashed) contributions to the electric current, $(c)$ density of free surface charge and $(d)$ axial forces acting on the inner liquid per unit streamwise length (electric force, $2 \pi r_{s} \tau_{f}^{*}$ in the last term of (2.8) (solid); force due to the axial viscous stress in the inner liquid, first term of (2.8) (dashed); force due to the normal electric stress and the surface tension, $\pi r_{s}^{2} \partial\left(\tau_{n}^{e}-1 / r_{s}\right) / \partial x$ in the second term of (2.8) (chain) and viscous force due to the outer liquid, $2 \pi r_{s} \tau_{t}^{0}$ in the last term of (2.8) (dotted)) as functions of the distance $x$ to the plate for $C a=3, E_{\infty}=2, \epsilon=20$, $A=10^{3}$ and $\lambda=0,0.01$ and 0.1 , increasing as indicated by the arrows.

not tend to zero far from the orifice when $\lambda>0$. As was discussed in $\$ 2$, this is due to the viscous shear stress of the outer liquid on the interface, which slows down the inner liquid and causes the jet to thicken in order to absorb the imposed dimensionless flow rate $C a$. The increase of the radius has a direct effect on the size of the drops, if the jet eventually breaks up. It also has an effect on the electric current transported by the jet. The bulk conduction current and the surface convection current are shown in figure $6(b)$. The conduction current does not tend to zero far downstream. When $\lambda$ increases, the conduction current increases and makes an increasing contribution to the total current, which also increases (see figure 1), while the convection current decreases. The density of electric charge at the interface (figure $6 c$ ) increases with $\lambda$, despite the decrease of $I_{s}=2 \pi \sigma v r_{s}$, because the decrease of $v r_{s}$ overcomes that of $I_{s}$. The continuous stretching of the jet that causes $\sigma$ to decrease downstream of the current transfer region when $\lambda=0$ ceases to exist when $\lambda>0$. The maximum of $\sigma$ becomes shallow and even disappears when $\lambda$ increases. The axial forces per unit length of the jet in the region of quasi-unidirectional flow are shown in figure $6(d)$. The electric force (solid curves) does not tend to zero far downstream when $\lambda>0$. It 

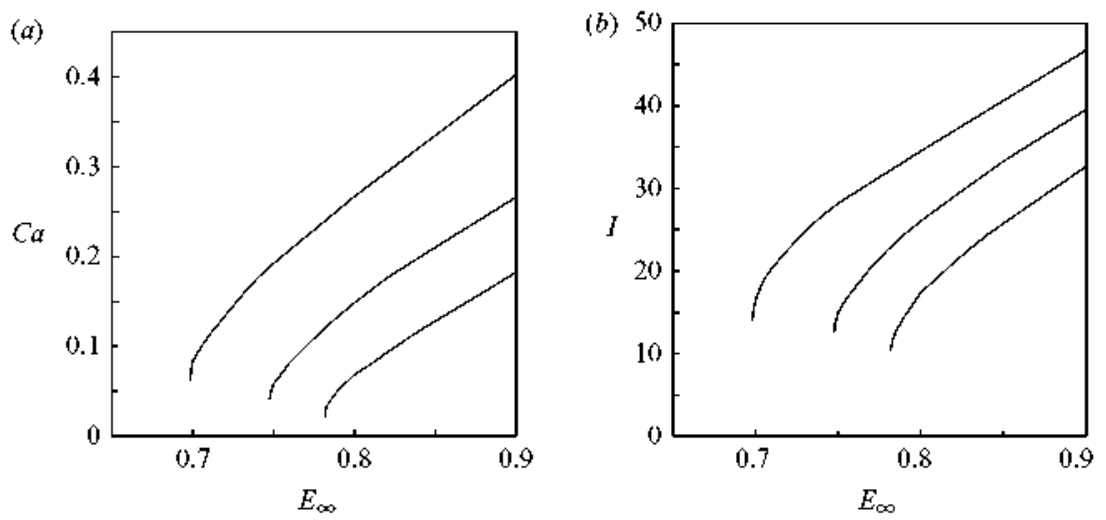

Figure 7. Contour of $V=0.9$ in the electric field/flow rate plane $(a)$ and the electric field/electric current plane (b) for $\epsilon=20, A=10^{3}$ and $\lambda=0,0.01$ and 0.03 , increasing from top to bottom.

is balanced mainly by the viscous force due to the outer liquid $\left(2 \pi r_{s} \tau_{f}^{0}\right.$ in $(2.8)$; dotted curves in figure $6 \dot{d}$ ), while the force due to the axial viscous stress of the inner liquid (dashed curves) tends to zero faster than when $\lambda=0$.

Apparently it is not always possible for the jet to attain the asymptotic viscouselectric shear balance discussed in $\$ 2$. The numerical computations fail to converge to a stationary solution above a certain $C a$ that decreases when $\lambda$ increases or $E_{x:}$ decreases, as was mentioned before. Notice that this limitation due to the viscous shear stress on the interface of the jet is different from the limitation due to the drag of the drops generated by the breakup of the jet, which leads to a space charge that reduces the field on the meniscus if the voltage applied between the electrodes is kept constant (Fernández de la Mora 2007).

The increase of the electric current with $\lambda$ in figure 1 is accompanied by an increase of the volume of the meniscus, whose shape also changes, making the transition to the jet more smooth when $\lambda$ increases. If the volume of the meniscus is kept constant, then the flow rate and the electric current are decreasing functions of $\lambda$ (see figure 7). The minimum value of $E_{00}$ at which a stationary solution with a jet exists for a given volume of liquid increases with $\lambda$.

The order of magnitude estimates leading to (3.1) can be extended to take into account the effect of the viscous shear of the outer liquid on the interface, and thus may shed some light on the conditions under which this effect is important. For very small values of $\lambda$, the effect of the outer liquid comes into play only far downstream of the current transfer region, at distances from the orifice where $r_{s}$ given by (2.9) becomes of the order of $r_{s \alpha}$ in (2.11) and the continuous stretching of the jet is arrested. The ratio of this asymptotic radius to the characteristic radius of the jet in the current transfer region $\left(r_{s T}\right.$ in (3.1)) is

$$
\frac{r_{s \alpha_{s}}}{r_{s_{T}}} \sim \Pi^{1 / 4} \text { with } \Pi=\frac{\lambda \Lambda^{1 / 2}}{E_{x_{i}}} .
$$

where $I$ from (3.1) has been used to evaluate $r_{s \infty 0}$. Thus, the conduction current left in the jet is small compared to the total current, and the estimates (3.1) apply, when $\Pi \ll 1$. When $\Pi$ is of order unity, the viscous shear stress of the outer liquid is of the order of the electric shear stress in the current transfer region, and the two stresses 
can balance each other before the transfer of current to the interface is complete. This result provides a qualitative explanation of the numerical computations, which show that the fraction of the total current left as conduction current in the far jet increases with $\lambda$ (figure $6 b$ ) and also when $A$ increases or $E_{x}$ decreases (results not displayed). The parameter group $G=I^{3} / \lambda A^{2} E_{i 0} C a^{2}$ in the discussion following (2.10) is large when $\Pi \ll 1$, in which case the radius of the jet approaches the lower root of (2.10) when the viscous shear stress of the outer liquid becomes important far from the orifice, while $G=O(1)$ when $\Pi=O(1)$, in which case the two positive roots of (2.10) are of the same order or do not exist.

The estimates also suggest that, when $\Pi$ becomes large, the axial force $2 \pi r_{s} \tau_{f}^{0}$ due to the viscous shear stress of the outer liquid becomes of the order of the axial viscous force of the inner liquid (first term of $(2.8)$ ) in a leading region of the jet where the electric current is still dominated by conduction in the inner liquid. The axial field induced by the surface charge of the jet almost completely balances the applied field in this region, making $E_{t} \ll E_{x, *}$ The surface charge satisfies $\sigma r_{s} / x \sim E_{x, \text {, }}$ as in the derivation of (3.1), so that $\tau_{n}^{e} \sim\left(x / r_{s}\right)^{2} E_{i \infty}^{2}$ and $\pi r_{s}^{2} \partial \tau_{n}^{e} / \partial x \sim E_{x x}^{2} x$ in the second term of (2.8). This force, due to the axial gradient of the pressure variation induced by the normal electric stress, drives the liquid in the region of interest, which is defined by the simultaneous balances $\partial\left(3 \pi r_{s}^{2} \partial v / \partial x\right) / \partial x \sim \pi r_{s}^{2} \partial \tau_{t /}^{e} / \partial x \sim 2 \pi r_{s} \tau_{t}^{0}$. Using $v \sim C a / r_{s}^{2}$ and $\tau_{t / 3}^{0} \sim \lambda v / r_{s}$, these balances give $x \sim \ell=C a^{1 / 3} / E_{\infty}^{2 / 3}$ (as in (3.1)) and $r_{s} \sim r_{s 0}=\lambda^{1 / 2} \mathrm{Ca}^{1 / 3} / E_{\infty}^{2 / 3}$. The term $\partial\left(3 \pi r_{s}^{2} \partial v / \partial x\right) / \partial x$ becomes negligible for $x \gg \ell$, and the balance of the other two terms gives then $r_{s} \sim \lambda^{1 / 2} C a^{1 / 2} / E_{\infty} x^{1 / 2}=r_{s 0}(\ell / x)^{1 / 2}$. The axial field needed in the inner liquid in order for conduction to supply the surface charge required in this region $\left(\sigma \sim\left(x / r_{s}\right) E_{\infty}\right)$ is $E_{1 x} \sim E_{\infty}^{5} x^{3} / \lambda^{2} \Lambda C a$, from the condition $A E_{1 x} r_{s}^{2} \sim \sigma v r_{s}$. This axial field becomes of the order of $E_{\infty}$ when $x \sim \lambda^{2 / 3} \Lambda^{1 / 3} \mathrm{Ca}^{1 / 3} / E_{\infty: 3}^{4 / 3}=\Pi^{2 / 3} \ell$, where $r_{s} \sim r_{s 0} / \Pi^{1 / 3}$ and $\left(I_{b}, I_{s}\right) \sim \lambda^{1 / 3} \Lambda^{2 / 3} E_{i \infty}^{1 / 3} \mathrm{Ca}^{2 / 3}$. The condition $E_{1 i} \sim E_{i 0}$ defines the current transfer region when $\Pi \gg 1$. The axial electric force $\left(2 \pi r_{s} \tau_{t}^{e}\right.$ in (2.8)) overcomes the pressure gradient force $\pi r_{s}^{2} \partial \tau_{h}^{e} / \partial x$ in the current transfer region, and the equilibrium $\tau_{t}^{0} \approx-\tau_{t}^{e}$ leading to $(2.10)$ is achieved downstream of this region. However, the estimate of the electric current derived here amounts to $G=O(1)$ for $\Pi \gg 1$, so that (2.10) might not have solution.

The role of the outer liquid discussed in this section can be easily played by a gas (which is very often present), given the small values of $\lambda$ required for the outer fluid to have a noticeable effect on the jet. The inertia of the outer fluid is not likely to bring qualitative changes to the results of this section insofar as the thickness of the viscous layer of this fluid is of the order of the radius of the jet or larger.

\section{Conclusions}

Numerical computations of the flow, the electric field and the transport of charge in the meniscus and the current transfer region of an electrospray of a very viscous liquid of small conductivity have been carried out to determine the conditions of existence of a stationary cone-jet. Different boundaries of the domain of operation in the applied field/flow rate plane have been found. At constant flow rate, the applied field can be varied between a minimum, below which the electric shear stress cannot sustain the cone-jet, and a maximum, above which the electric stress disrupts the meniscus. Both limits depend on the flow rate. Electric relaxation effects may be small in the current transfer region of the jet, which is a slender region for any value of the flow rate. 
A viscous dielectric fluid often surrounds the cone-jet, and the viscous drag due to this fluid then balances the electric shear force and stops the stretching of the jet at some distance from the meniscus. An estimate has been worked out of the conditions under which the effect of the outer liquid comes into play in the current transfer region and modifies the electric current. The numerical results show that a stationary cone-jet can be established only below a certain maximum flow rate in the presence of an outer fluid.

The author is indebted to Professor Fernández de la Mora (Yale) for insightful comments and criticisms. This work was supported by the Spanish Ministerio de Educación y Ciencia through Project No. DPI2007-66659-C03-02.

\section{REFERENCES}

Ashley, H. \& Landahl, M. 1965 Aerodynamics of Wings and Bodies, Ch. 6. Addison-Wesley.

Barrero, A., López-Herrera, J. M., Bouctard, A., Loscertales, I. G. \& Márquez, M. 2004 Steady cone-jet electrosprays in liquid insulator baths. J. Colloid Interface Sci. 272, 104108.

BATCHeLOR. G. K. 1967 An Introduction to Fluid DynamicsCambridge University Press, p. 132.

CHEN, D.-R. \& PUI, D. Y. H. 1997 Experimental investigation of scaling laws for electrospraying: dielectric constant effects. Aerosol Sci. Technol. 27, 367-380.

Cherney, L. T. 1999 Structure of Taylor cone-jets: limit of low flow rates. J. Fluid Mech. 378, $167-196$.

Cloupeau, M. \& Prunet-Foch, B. 1989 Electrostatic spraying of liquid in cone-jet mode. J. Electrost. 22, 135-159.

Cloupeau, M. \& Prunet-Foch, B. 1990 Electrostatic spraying of liquid. Main functioning modes. J. Electrost. 23, 165-184.

Cloupeau, M. \& Prunet-Foch, B. 1994 Electrohydrodynamic spraying functioning modes: a critical review. J. Aerosol Sci. 25, 1021-1036.

FENG, J. J. 2002 The stretching of an electrified non-Newtonian jet: a model for electrospinning. Phys. Fluids 14, 3912-3926.

Fernández de LA MORA. J. 1992 The effect of charge emission from electrified liquid cones. J. Fluid Mech. 243, 561-573.

FERnÁndeZ DE LA MORA, J. 2007 The fluid dynamics of Taylor cones. Amut. Rev. Fluid Mech. 39, $217-243$.

Fernández de la MoRa, J. \& LOSCertales, I. G. 1994 The current emitted by highly conducting Taylor cones. J. Fluid Mech. 260, 155-184.

Gañán-Calvo, A. M., Dávila. J. \& Barrero. A. 1997 Current and drop size in the electrospraying of liquids. Scaling laws. J. Aerosol Sci. 28, 249-275.

Guerrero, I.. Bocanegra, R., Higuera, F. J. \& Fernández de la Mora. J. 2007 Ion evaporation from Taylor cones of propylene carbonate mixed with ionic liquids. J. Fluid Mech. 591, 437-459.

HAPPEl, J. \& BRENNER, H. 1965 Low Reynolds Number Hydrodynamics. Prentice-Hall.

Hayati, I. Bailey, A. I. \& Tadros, TH. F. 1987 Investigations into the mechanisms of electrohydrodynamic spraying of liquids. J. Colloid Interface Sci. 117, 205-221.

Higuera. F. J. 2003 Flow rate and electric current enitted by a Taylor cone. J. Fluid Mech. 484 , $303-327$.

Higuera. F. J. 2006 Stationary viscosity-dominated electrified capillary jets. J. Fluid Mech. $\mathbf{5 5 8}$, $143-152$.

HIGUERA, F. J. 2007 a Stationary coaxial electrified jet of a dielectric liquid surrounded by a conductive liquid. Phys. Fluids 19, 012102.

Higuera, F. J. $2007 \mathrm{~b}$ Emission of drops from the tip of an electrified jet of an inviscid liquid of infinite electrical conductivity. Phys. Fluids 19,072113.

HigueRA, F. J. 2008 a Breakup of a supported drop of a viscous conducting liquid in a uniform electric field. Phys. Rev. E 77, 016314. 
Higuera, F. J. $2008 b$ Model of the meniscus of an ionic-liquid ion source. Phys. Rev. E 77, 026308. HINCH. E. J. 1991 Perturbation Methods. Cambridge University Press.

JAworek, A. \& Krupa, A. 1999 Classification of the modes of EHD spraying. J. Aerosol Sci. 30, $873-893$.

Kim, K. \& TuRnbull, R. J. 1976 Generation of charged drops of insulating liquids by electrostatic spraying. J. Appl. Phys. 47, 1964-1969.

LANDAU, L. D. \& Lifshitz, E. M. 1960 Electrodynamics of Continious Media. Pergamon.

LARriba. C. \& Fernández de LA Mora, J. 2009 Electrospraying insulating liquids via charged nanodrops injection from the Taylor cone of an ionic liquid. Phys. Fluids. (submitted).

Miksis, M. J. 1981 Shape of a drop in an electric field. Phys. Fluids 24, 1967-1972.

Pantano, C., Gañán-Calvo, A. M. \& Barrero, A. 1994 Zeroth order, electrohydrostatic solution for electrospraying in cone-jet mode. J. Aerosol Sci. 25, $1065-1077$.

Rosell-Llompart, J. \& Fernández de la Mora, J. 1994 Generation of monodisperse droplets 0.3 to $4 \mu \mathrm{m}$ in diameter from electrified cone-jets of highly conducting and viscous liquids. J. Aerosol Sci. 25, 1093-1119.

SAville, D. A. 1997 Electrohydrodynamics: the Taylor-Melcher leaky dielectric model. Anni. Rev. Fluid Mech. 29, 27-64.

SMITH. D. P. H. 1986 The electrohydrodynamic atomization of liquids. IEEE Trans. Ind. Appl. IA22, $527-535$.

TAYLOR, G. I. 1964 Disintegration of water drops in an electric field. Proc. R. Soc. Lond. A 280, 383-397.

Wohlhuter, F. K. \& Basaran, O. A. 1992 Shapes and stability of pendant and sessile drops in an electric field. J. Fluid Mech. 235, 481-510.

ZELENY, J. 1915 On the conditions of instability of electrified drops, with applications to the electrical discharge from liquid points. Proc. Camb. Phil. Soc. 18, 1-6.

ZELENY, J. 1935 The role of surface instability in electrical discharges from drops of alcohol and water in air at atmospheric pressure. J. Franklin Inst. 219, 659-675. 